\title{
Tophi reduction: ultrasound imaging and correlation with plasma levels of uric acid in patients undergoing treatment for tophaceous gout
}

\author{
A.J.L. Ferrari ${ }^{1}$, A.R. Corrêa Fernandes ${ }^{2}$, R. de Almeida Agustinelli', \\ H. Seike ${ }^{2}$, E. de Ávila Fernandes ${ }^{2}$ \\ ${ }^{1}$ Discipline of Rheumatology, Medical Department, EPM-UNIFESP, São Paulo, Brazil; \\ ${ }^{2}$ Department of Diagnostic Imaging, EPM-UNIFESP, São Paulo, Brazil
}

\begin{abstract}
SUMMARY
The objective was to determine the reduction of tophi in patients undergoing drug therapy in correlation with urate serum levels through ultrasound examination.

A total of 31 male patients, between the ages of 33 to 77 years, with tophaceous gout were evaluated between 2005 and 2009, 11 of which were selected. Ultrasound examinations of visible tophi and evaluations of serum uric acids levels were performed annually on each patient.

There was a statistically significant difference between measurements 1, 2, 3 and measurement 4 and between measurements 4 and 5. A strong significant positive association was seen between variation of tophus size and the reduction of serum uric acid levels.
\end{abstract}

Key words: Gout; ultrasonography; uric acid.

Reumatismo, 2019; 71 (2): 75-80

\section{INTRODUCTION}

out is metabolic disease whose clini-

Jcal manifestations are related to the deposit of monosodium urate crystals in various tissues. When serum uric acid levels are elevated and exceed saturation $\mathrm{pH}$, the uric acid transforms into crystal and is deposited in the tissue (1).

The clinical course of the disease shows periods of arthritis with intermittent asymptomatic periods, known as intercritical periods. In the first stages of the disease, the arthritis is monoarticular and in chronic phases it is oligoarticular or polyarticular of incomplete resolution, with reduction or complete absence of the intercritical period (1).

The presence of tophi is due to inadequate treatment or untreated disease and incorporates monosodium urate crystal deposits, protein matrix and inflammatory cells in tissues (1). Generally, manifestation of tophi occurs between 5 and 10 years of inadequately treated disease, according to literature. The tophi may be deposited in tendons, ligaments, cartilage, bones and soft tissue, including synovial bursae and pockets, enabling the formation of deformities (1).

Treatment of gout includes the reduction of urate levels, accomplished with the use of synthesis inhibitors (allopurinol, febuxostat) and uricosuric agents (benzbromarone). Allopurinol acts on the xanthine oxidase enzyme, inhibiting the synthesis of uric acid while febuxostat is a non-purine selective inhibitor of santhine oxidase enzyme. Benzbromarone is a uricosuric drug, which reduces the level of uric acid through its increased excretion (2/3 via intestinal and 1/3 via renal).

The objective in uric acid reduction therapy is to dissolve the monosodium urate crystals (MSU) crystals when a concentration lower than serum urate saturation is reached $\overline{\text { Corresponding author: }}$ Renan de Almeida Agustinelli Universidade Federal de São Paulo, Rua Botucatu 740

$3^{\text {rd }}$ Floor, Rheumatology Division São Paulo 04023-062, Brazil E-mail: re_augusto@yahoo.com.br 
$(2,3)$. The subsaturated serum urate has been associated with the reduction, and ultimately the dissappearance, of tophi. The inversely proportional correlation between the uric acid and the pace of reduction of the tophi has been documented $(2,4)$.

Ultrasonographic evaluation is an important and non-invasive tool able to help in several aspects of gout, such as differential diagnosis, identification of MSU crystal deposit and monitoring therapeutic response. Some findings, such as double contour sign, inhomogeneous material surrounded by a small anechoic rim related to the presence of tophy, and erosions, may be identified by ultrasonography (5-7).

There are few studies in the literature, which use ultrasound follow-up in the evaluation of tophi (2). Ultrasound, which is an easily performed process, is clearly an important tool in the evaluation of tophi during the course of drug therapy for the reduction of gouty tophi.

\section{Objectives}

To determine the reduction of tophi in patients with gout undergoing drug therapy; to verify the correlation between the maintenance of appropriate plasma urate levels, that is, below normal/safe values, and the reduction of gout tophi using ultrasound as an imaging method in the evaluation and follow up of tophi measurement.

\section{MATERIALS AND METHODS}

A prospective observational study was approved by the Medical Ethics Committee at UNIFESP (protocol \#1580/06), where the study was carried out. All patients were sent consecutively from the Microcrystal Disease Ambulatory of the Discipline of Rheumatology of UNIFESP and volunteered to take part in this study; they all signed a free and informed consent form.

Thirty-one male patients ranging from 33 to 77 years old (mean age 54.7) were examined between October 2005 and April 2009. They were diagnosed with tophaceous gout by the presence of MSU, identified using an optical microscope with polarized light on aspirated synovial fluid or by meeting the Wallace criteria (8) or the EULAR recommendations for the diagnosis of gout (9).

Patients who did not present visible signs of tophi were excluded, as were patients who did not have sufficient follow-up of tophi evolution, patients who were unsuitable for treatment, and lastly, patients with other crystal deposition diseases. Of the 31 patients, 11 were selected between the ages of 33 and 77 (mean 52.25 years) who were undergoing drug therapy for the reduction of serum urate levels using allopurinol in doses varying between $100 \mathrm{mg}$ and $600 \mathrm{mg}$ per day and benzbromarone in doses vaying between $50 \mathrm{mg}$ and $300 \mathrm{mg}$ per day.

Dynamic ultrasound exams of visible tophi were carried out on each patient, independently of consistency, soft and/or hardened, using a Sonoline Antares (Siemens Medical Solutions, Germany), with a high-resolution linear transducer (frequency range: $8 \mathrm{MHz}$ to $14 \mathrm{MHz}$ ) and an Envisor (Philips Medical Systems, Bothell, USA) with a high-resolution linear transducer (frequency range: 3 $\mathrm{MHz}$ to $12 \mathrm{MHz}$ ). The exams were carried out in the Ultrasound Sector of the Diagnostic Imaging Department of the Univesidade Federal de São Paulo - UNIFESP by a radiologist with 15 years' experience in musculoskeletal ultrasonography.

Specific parameters were used for the study of superficial structures, with equipment optimized for musculoskeletal examination. Extended images were obtained in areas with accentuated curvature under the skin in order to facilitate and obtain more precise measurements. Gel was used to provide a good acoustic window and avoid interpositioning of air between the transducer and the skin.

The findings were studied and documented in the longitudinal and transversal planes for an adequate characterization of the lesions' limits and adequate gauging. The contralateral side was examined for a comparison with the normal whenever possible. Calculation of tophi volume was carried out using software of the same equipment utilizing the formula $(\mathrm{AP} \times \mathrm{CC} \times \mathrm{LL} \times 0.523)$ used to calculate ovoid volumes, considered adequate for the evaluation. 
Annual evaluation and laboratory examinations were carried out on the tophi, including serum urate, on dates close to randomized consultations of patients who were undergoing drug therapy.

The volume measurements of the tophi were tabulated in order to ascertain whether there had been any alterations such as increase, decrease and/or stabilization of tophi volume. The statistical analysis of tophi volume, obtained by measurement during the follow-up period, was as follows.

The data were shown in tables and graphs. The Shapiro-Wilk test was used to verify normality of the data.

The descriptive statistic consisted of calculation of average and standard deviation for all continuous and semi-continous data with normal distribution. The categorical data were expressed in absolute and relative frequency. In the graphs referring to comparisons of measurements, a confidence interval of $95 \%$ (CI 95\%) of the average was used; when dealing with repeated measurements the CI 95\% was calculated.

For the comparison of tophus size at dif-

Table I - Descriptive data of evaluated sample characterization.

\begin{tabular}{|c|c|c|}
\hline \multicolumn{3}{|c|}{ Variable } \\
\hline \multicolumn{2}{|c|}{ Age (years) } & 52.2 \\
\hline \multicolumn{2}{|c|}{ Duration of disease } & $11+5.81$ \\
\hline \multicolumn{2}{|c|}{ Initial uric acid (mg/dL) } & $8.39+1.23$ \\
\hline \multicolumn{2}{|c|}{ Final uric acid (mg/dL) } & $4.92+2.55$ \\
\hline \multicolumn{2}{|c|}{ Follow-up time (years) } & $3.79+0.73$ \\
\hline \multirow[t]{2}{*}{ ESRD } & Yes & $2(18.2 \%)$ \\
\hline & No & $9(81.8 \%)$ \\
\hline
\end{tabular}

ESRD, end stage renal disease. Data represented in mean + standard deviation or absolute frequency (relative frequency \%). ferent times an ANOVA, a parametric test for repeated measurements, was used. The Wilcoxon non-parametric test was also used.

Correlation was considered as follows: 0.00 to 0.19 - very weak, 0.20 to 0.39 - weak, 0.40 to 0.69 - moderate, and 0.70 to 0.89 - very strong. Input Landis and Koch referrence.

A value of a $p \leq 0.05$ was considered as statistically significant.

To facilitate the visualization of level of significance, the following was used: $*=\mathrm{p}<0.01 ; * * *=\mathrm{p}<0.001$.

For the analyses, the SPSS statistical software 17.0 for Windows (SPSS Inc.) and Prism 5.0 for Windows (GraphPad Software Inc.) were used (10-18).

To observe the variation in tophi size in patients over time, a percentage relative to the initial size of tophus was considered (measurement 1) so that tophi of different areas could be compared without great influence to the measurement. In other words, all tophi were considered as $100 \%$; if in the first measurement. For example; if a tophus on measurement 1 was $3 \mathrm{~cm}$, this was considered $100 \%$, if on measurement 2 it measured $1 \mathrm{~cm}$ it would be computed as $33.33 \%$ as it presented $1 / 3$ of the initial value.

\section{RESULTS}

The patient's data is shown in Tables I and Table II shows alteration in gout tophi size. Figure 1 presents the data regarding change in tophus size during the follow-up period. Measurements 1, 2 and 3 were statistically similar and there was a statistically significant reduction between measurements 1,2 , 3 and measurement 4 and between measurement 4 and measurement 5 . Therefore it

Table II - Data of tophi size $(n=56)$ evaluated in 11 patients.

\begin{tabular}{|l|l|c|c|c|c|c|}
\hline \multicolumn{2}{|c|}{} & Measure 1 & Measure 2 & Measure 3 & Measure 4 & Measure 5 \\
\hline \multirow{2}{*}{$\begin{array}{l}\text { Actual size } \\
\text { (cm) }\end{array}$} & Mean & 2.12 & 2.23 & 2.47 & 1.87 & 1.32 \\
\cline { 2 - 7 } & SD & 2.36 & 2.38 & 3.49 & 2.94 & 1.93 \\
\hline \multirow{2}{*}{$\begin{array}{l}\text { \% in relation } \\
\text { to initial size }\end{array}$} & Mean & 100.00 & 161.42 & 163.90 & 89.27 & 77.09 \\
\cline { 2 - 7 } & Cl 95\%* & $77.75-122.24$ & $111.37-211.45$ & $124.01-203.79$ & $65.47-113.07$ & $50.72-103.45$ \\
\hline
\end{tabular}

SD, standard deviation; Cl, confidence interval. *As proposed by Loftus/Masson (1994). 


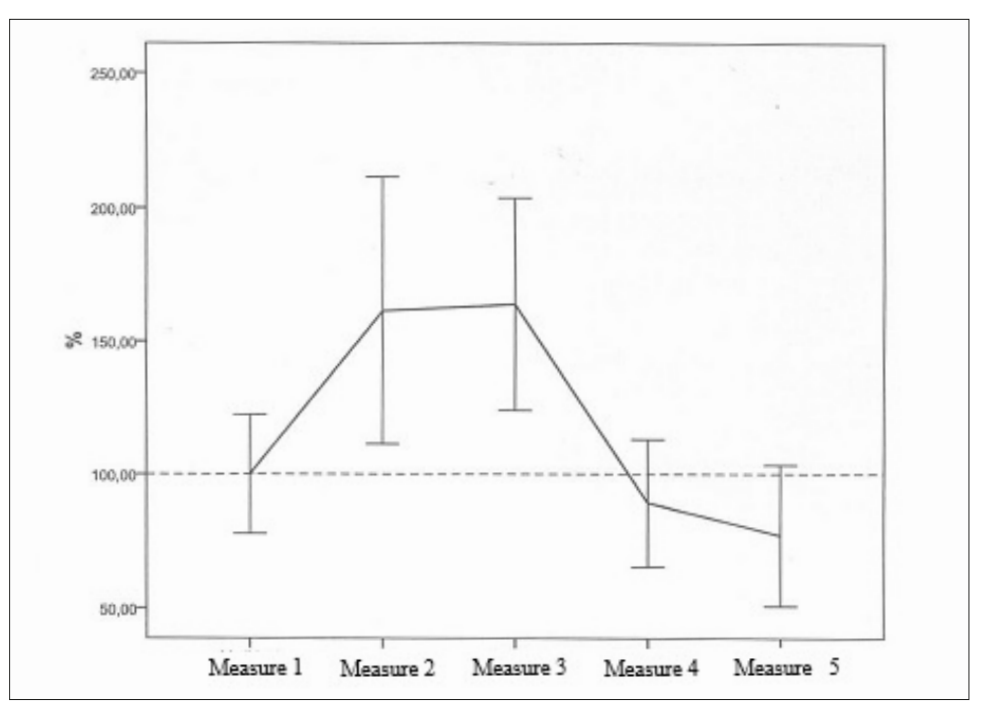

Figure 1 - Relative variation of tophi size during study ( $n=56)$.

can be concluded that there was a reduction in tophi starting from measurement 4 . And, with the ANOVA test, measurements 1,2, 3 and 4 were statistically similar, the tophi reduction occured from measurement 5 . Both tests showed reduction in tophi, from measurements 4 and 5 respectively.

This fact is perfectly compatible with clinical results, when a reduction in tophus is verified after a period of regular and daily drug therapy.

A correlation between the maintenance of adequate serum urate, that is to say reduc-

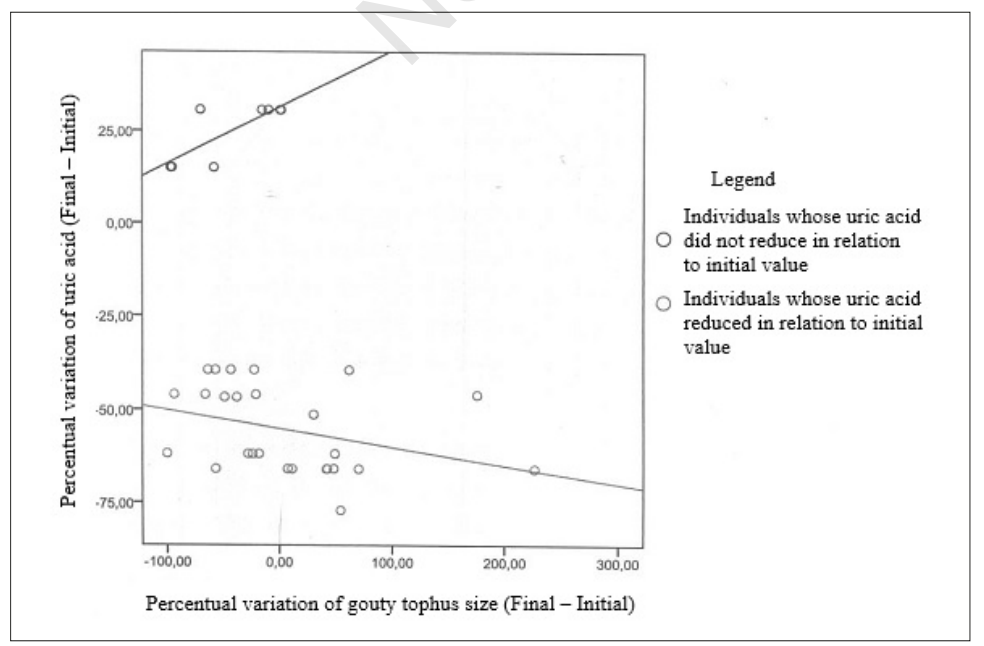

Figure 2 - Association between percentual variation of tophus size and percentual variation of serum urate $(n=34)$. tion of uric acid levels to below the normal (>7 mg/dL in males) and/or safe levels, and the reduction of tophi volume in patients was verified (Figure 2).

With the ANOVA (parametric) test with repeated measurements, regarding $\mathrm{p}$ values, the results were: measurement $1=$ measurement $2=$ measurement $3>$ measurement 5. In summary, a statistical reduction of tophi was detected only in measurement 5 when compared with measurements 1,2 and 3 . The average value in measurement 4 is statistically equal to the values of all other times, or rather, measurement 4 is statistically equal to all other measurements.

From a statistical point of view it is possible to affirm that the tophi reduced significantly in size in relation to their size at the start of the study.

Table III shows the plasma urate levels and the presence or absence of chronic renal failure.

Uric acid was measured at the same time as tophi were evaluated by ultrasound.

The negative values represent a reduction in relation to the initial values and positive values represent an increase in relation to initial values (for tophus size as well as urate value).

An exploratory graph analysis shows that patients who presented a reduction in urate levels during treatment presented an alteration in tophus size different from those who did not show the same reduction. This made it possible to analyse adequately these two subgroups separately. Figure 2 shows the association of size variation in relation to the variation of serum urate and its behaviour in the two aforementioned subgroups. Ultrasound images of the evolution of tophus size in one patient can be observed in Figure 3.

\section{DISCUSSION}

Over the last years there has been growing interest in the use of ultrasound in rheumatology. The advantages include the absence of radiation, low cost, patient cooperation, dynamic multi-plane images, high resolution and an effective method of guiding invasive procedures. 
Table III - Values in $\mathrm{mg} / \mathrm{dL}$ of serum urate and concomitance of end stage renal disease.

\begin{tabular}{|c|c|c|c|c|c|c|}
\hline \multirow{2}{*}{ Patients } & \multirow{2}{*}{ ERSD } & \multicolumn{7}{|c|}{ Uric acid mg/dL } \\
\cline { 3 - 7 } & & $\mathbf{1}$ & $\mathbf{2}$ & $\mathbf{3}$ & $\mathbf{4}$ & $\mathbf{5}$ \\
\hline 1 & No & 9.4 & 5.4 & 6.5 & 3.6 & - \\
\hline 2 & No & 7 & 3 & 2.6 & 7.4 & 2.4 \\
\hline 3 & No & 7.2 & 3.5 & 5.9 & 10.7 & 5.3 \\
\hline 4 & No & 8 & 5.8 & 3.3 & 3.9 & - \\
\hline 5 & Yes & 7.6 & 10.3 & 4 & 4.8 & 4.1 \\
\hline 6 & No & 11.1 & 6.2 & 10 & 6.7 & 3.8 \\
\hline 7 & Yes & 8 & 3.1 & 6.2 & 9.2 & - \\
\hline 8 & No & 8.9 & 6.6 & 5 & 6 & 4.8 \\
\hline 9 & No & 7.7 & 6.2 & 5.1 & 3.2 & 4.1 \\
\hline 10 & No & 7.8 & 8.7 & 6.8 & 5.8 & 10.2 \\
\hline 11 & No & 9.6 & 2.6 & 4.3 & 3.2 & 2.2 \\
\hline
\end{tabular}

ESRD, end stage renal disease.

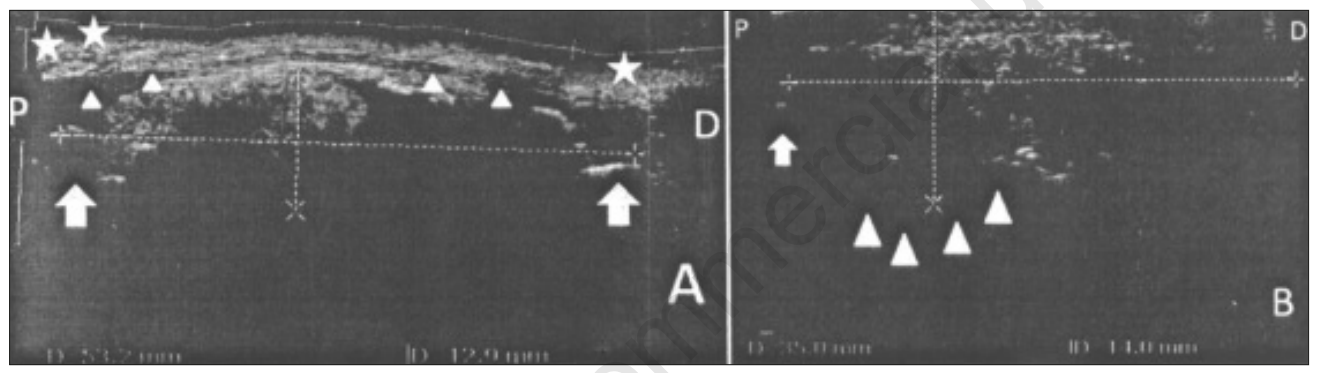

Figure 3 - Ultrasound images in the longitudinal planes ( $p$ proximal and $d$ distal) of tophi located on the first right metatarsophalangeal articulation. The largest axes of the tophi are indicated between the dotted lines and Xs. The arrows mark the cortical bone of the first metatarsal and the proximal phalanx of the hallux (*marks the subcutaneous tissue and skin). A) The tophus in the first evaluation of the posterior structures. The tophus dislocates the extensor tendon of the hallux (arrowheads). B) The evolution of tophus reduction after 9 months of therapy with allupurinol and benzbromarone is demonstrated by the measurements at the bottom of the images. After therapy the calcifications deteriorated and the posterior outline of the tophus is seen, now clearly showing erosions to the bone of the first metatarsal (arrowheads).

The physics of ultrasound makes it an ideal tool for the detection of crystaline material in soft tissue. It has long been used in the detection of biliary and urinal calculi, as ultrasound visualizes tissues as acoustic reflections and the crystaline material found in the articulations in cases of gout reflects the sound waves with higher intensity than adjacent tissues such as hyaline cartilage and synovial fluid (19).

Other methods of imaging may be used in the follow-up of patients with gouty tophi throughout the serum urate reduction therapy. However, not only do they not have all the aforementioned advantages, they also have some disadvantages, such as the use of ionizing radiation, cost and restricted access.

Despite the difficulty of finding comprehensive studies on this topic in the literature, the study by Perez-Ruiz et al. (2) shows the validity of ultrasound in the measurement of tophi while carrying out therapeu- 
tic reduction of serum urate, evaluating 50 articulations of 23 patients, comparing the ultrasound results with magnetic resonance. A good correlation was shown between the imaging methods as much in sensitivity and specificity as in correlation between measurements and volume of tophi.

Therefore, as an acceptable follow-up method for tophi evaluation, ultrasound was used throughout the period of serum urate reduction treatment.

Serum urate reduction treatment has proven to be a good therapeutic instrument in maintaining volume of tophi caused by gout and the follow-up of the volume of these tophi can be assessed by ultrasound, which is a valid, quick and low-cost technique.

\section{CONCLUSIONS}

This study shows the reduction of tophi in patient follow-up and the existence of a relationship between the maintenance of adequate plasma uric acid levels and the reduction in volume of tophi using ultrasound imaging as an effective method of evaluation. It has managed to establish a statistically significant relation between the reduction of tophi volume and the serum urate level.

\section{REFERENCES}

1. Monu JU, Pope TL Jr. Gout: a clinical and radiologic review Radiol Clin North Am. 2004; 42: 169-84.

2. Perez-Ruiz F, Martin I, Canteli B. Ultrasonographic measurement of tophi as na outcome measure for chronic gout. Rheumatol. 2007; 34: 1888-93.

3. Perez-Ruiz F, Calabozo M, Fernandez-Lopez MJ, et al. Treatment of chronic gout inpatients with renal function impairment. An open, randomized, actively controlled study. J Clin Rheumatol. 1999; 5: 49-55.

4. Perez-Ruiz F, Calabozo M, Pijoan JI, et al. Effect of uratelowering therapy on the velocity of size reduction of tophi in chronic gout. Arthritis Rheum. 2002; 47: 356-60.
5. Machado PM, Koevoets R, Bombardier C, van der Heijde DM. The value of magnetic resonance imaging and ultrasound in undifferentiated arthritis: a systematic review. J Rheumatol Suppl. 2011; 87: 31-7.

6. Naredo E, Uson J, Jiménez-Palop M, et al. Ultrasound-detected musculoskeletal urate crystal deposition: which joints and what findings should be assessed for diagnosing gout? Ann Rheum Dis. 2014; 73: 1522-8.

7. Thiele RG. Role of ultrasound and other advanced imaging in the diagnosis and management of gout. Curr Rheumatol Rep. 2011; 13: 146-53.

8. Wallace SL, Robinson H, Mais AT, et al. Preliminary criteria for the classification on the acute arthritis of primary gout. Arthritis Rheum 1977; 20: 895-900.

9. Zhang W, Doherty M, Pascual E, et al. EULAR evidence based recommendations for gout. Part I: Diagnosis. Report of a task force of the Standing Committee for International Clinical Studies Including Therapeutics (ESCISIT). Ann Rheum Dis. 2006; 65: 1301-11.

10. Berquó ES, Souza JMP, Gotlieb SLD. Bioestatística. São Paulo: E.P.U.; 1981. 350 p.

11. Dytham C. Choosing and using statistics: a biologist's guide Calvin Dytham. Oxford: Blackwell Science; 1999. 218 p.

12. Fletcher RH, Fletcher SW, Wagner EH. Trad. Maria Inês Schimidt. Epidemiologia Clínica: elementos essenciais. E.ed. Porto Alegre: Artes Médicas; 1996. 281 p.

13. Graphpad Software. GraphPad Prism 5.0: Learning Guide. Available from: http://www. graphpad.com/help/prism5/prism5help.html Accessed: 20 Jan, 2008.

14. Jekel JF, Katz DL, Elmore JG. Trad. Ricardo Savaris. Epidemiologia, bioestatística e medicina preventiva. Porto Alegre, RS: Artes Médicas; 2002. 328 p.

15. Shimakura SE. Laboratório de estatística e Geoinformação, UFPR, Brasil, Jan, 2007. Available from: http://leg.ufpr.br/ shimakur/ Accessed: 15 Nov, 2007.

16. Siegel S. Estatística não-paramétrica para as ciências do comportamento. São Paulo: Makron Books; 1975; 350 p.

17. Vieira S. Bioestatística: Tópicos avançados. 2. Ed. Rio de Janeiro: Campus; 2003; 216 p.

18. Vieira S. Introdução à Bioestatística. 2.ed. Rio de Janeiro: Campus; 1991; 203 p.

19. Thiele RG, Schlesinger N. Diagnosis of gout by ultrasound. Rheumatology (Oxford) 2007; 46: 1116-21. 